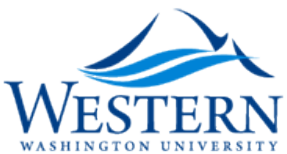

Western Washington University Western CEDAR

2016

\title{
Body Weight, Marital Status, and Changes in Marital Status
}

Jay Teachman

Western Washington University, jay.teachman@wwu.edu

Follow this and additional works at: https://cedar.wwu.edu/sociology_facpubs

Part of the Sociology Commons

\section{Recommended Citation}

Teachman, Jay, "Body Weight, Marital Status, and Changes in Marital Status" (2016). Sociology. 15.

https://cedar.wwu.edu/sociology_facpubs/15

This Article is brought to you for free and open access by the Social and Behavioral Sciences at Western CEDAR. It has been accepted for inclusion in Sociology by an authorized administrator of Western CEDAR. For more information, please contact westerncedar@wwu.edu. 


\title{
Body Weight, Marital Status, and Changes in Marital Status
}

\section{Jay Teachman'}

\begin{abstract}
In this article, I use 20 years of data taken from the 1979 National Longitudinal Study of Youth to examine the relationship between body weight and both marital status and changes in marital status. I use a latent growth curve model that allows both fixed and random effects. The results show that living without a partner, either being divorced or never married, is associated with lower body weight. Cohabitors and married respondents tend to weigh more. Marital transitions also matter but only for divorce. Gender does not appear to moderate these results.
\end{abstract}

\section{Keywords}

marriage, body weight, growth curve model, longitudinal, gender

Body weight and health are closely linked. Heavier individuals are more likely to suffer from a number of chronic conditions such as diabetes, cardiovascular disease, and some types of cancer (Bray, 2004; Ferraro \& KelleyMoore, 2003; Hill, Wyatt, Reed, \& Peters, 2003; Must et al., 1999; Thompson, Edelsberg, Colditz, Bird, \& Oster, 1999). Disparities in chronic health associated with excess body weight are in turn linked to higher rates of mortality (Breeze, Clarke, Shipley, Marmot, \& Fletcher, 2006). An appreciation of the factors linked to body weight is therefore an important component of public

IWestern Washington University, Bellingham, WA, USA

\section{Corresponding Author:}

Jay Teachman, Department of Sociology, Western Washington University, 516 High Street, Bellingham, WA 98225-908I, USA.

Email: jay.teachman@wwu.edu 
health. In this article, I build on prior research to investigate the role that marital status and changes in marital status play in determining body weight. I use a latent growth curve model (LGCM) that allows fixed effects to assess multiple measures of the body mass index (BMI) of respondents taken from the 1979 National Longitudinal Survey of Youth (NLSY). I also examine whether gender moderates the relationship between BMI, marital status, and marital transitions.

\section{Prior Literature on Marital Status, Marital Transitions, and Body Weight}

Early literature on the topic generally found that marriage is positively linked to body weight, whereas being divorced and widowed are negatively related to body weight (Hahn, 1993; Jeffrey \& Rick, 2002; Meltzer \& Everhart, 1995; Sobal, 1991; Sobal, Rauschenbach, \& Frongillo, 1992). Other early research found that changes in marital status are also related to body weight (French et al., 1994; Kahn \& Williamson, 1990, 1991; Rauschenbach, Sobal, \& Frongillo, 1995; Umberson, 1992). Importantly, however, most of the early research failed to make a conceptual distinction between marital status and changes in marital status, either because of conceptual limitations or data limitations. Thus, this early literature does not provide clear guidance about the relative importance of statuses versus transitions.

Although some current research continues to focus on either the relationship between body weight and marital status (Averett, Argys, \& Sorkin, 2013; Shafer, 2010), or between body weight and marital transitions (Harris, Lee, \& DeLeone, 2010; Sobal, Rauschenbach, \& Frongillo, 2003; The \& GordonLarson, 2009), a number of studies have begun to argue the importance of separating the effects of marital status from changes in marital status (Averett, Sikora, \& Argys, 2008; Umberson, Liu, \& Powers, 2009; Wilson, 2012). The distinction is important both theoretically and empirically. Theoretically, different processes are thought to link marital status and transitions in marital status to body weight. Empirically, the failure to distinguish between marital statuses and marital transitions leads to biased estimates of the effects of each, thus hindering attempts to discover the theoretical mechanisms linking marriage, divorce, cohabitation, and other living arrangements to body weight.

In this article, I unify the prior literature by using more than 20 years of data on BMI and marital status taken from the 1979 NLSY. I examine the joint effects of both current marital status and transitions in marital status. I also consider whether gender moderates the relationship between body weight, marital status, and marital transitions. I conduct my analyses using a LGCM that incorporates both fixed and random effects. 


\section{Why Should Marital Status Be Related to Body Weight?}

There are three major perspectives linking body weight to marital status. The first perspective, the resource model, emphasizes different resources, social and economic, available to individuals possessing different marital statuses (Waite, 1995). Most important, perhaps, married individuals are more likely to have a confidant with whom to eat and may therefore eat more regularly, leading to weight gain (Averett et al., 2008, 2013; Jeffrey \& Rick, 2002; Umberson et al., 2009; Wilson, 2012). Married individuals are also less likely to smoke, which may also act to increase body weight because smoking, which suppresses appetite, may lead to lower body weight (Flegal, Troianao, Pamuk, Kuczmarski, \& Campbell, 1995).

The second model, the attractiveness model, links body weight to differences in emphasis people place on their physical attractiveness. Married men and women are less likely to be conscious of or concerned about their body weight because they are not actively seeking a mate (Averett et al., 2008, 2013; Averett \& Korenman, 1996, 1999; Carmalt, Cawley, Joyner, \& Sobal, 2008; Shafer, 2010; Umberson et al., 2009; Wilson, 2012). As a consequence, married individuals are more likely to experience greater increase in body weight than comparable nonmarried individuals who are attempting to minimize weight gain in order to attract a partner.

The attractiveness model is also the basis on which some researchers expect that marital status and marital status transitions are more important for women than men (Averett et al., 2008; Harris et al., 2010; Sobal et al., 2003; The \& Gordan-Larsen, 2009; Wilson, 2012). This expectation is based on the belief that physical attraction is more important in the marriage market for women than for men (Averett \& Korenman, 1996; Conley \& Glauber, 2005). In addition, other research showing that women are more likely to take care of men rather than the reverse suggests that the marriage market effect is more important for women (Averett et al., 2008; Umberson et al., 2009).

The third model, the crisis model, focuses on stresses associated with change in marital status, particularly marital dissolution (via divorce or death). Stresses linked to marital disruption have been related to psychological, physiological, and social consequences that can lead to weight loss (Greeno \& Weng, 1994; Umberson et al., 2009; Williams, 2003; Williams \& Umberson, 2004). Unlike the case for the resource or attractiveness models, however, the crisis model predicts short-term or transitory effects of a crisis on body weight (Wilson, 2012). That is, the effects of marital transitions are expected be relatively short-lived, because after a crisis, individuals are expected to adjust to their new social and economic environment, making the effect of a transition transitory. 
The transitory effects in body weight associated with the crisis model suggest that there should be relatively small or nonexistent effects on subsequent health, because body weight will return to its static level (Wilson, 2012). Only more permanent shifts in body weight associated with the attractiveness or resource models should register subsequent effects on health. It is for this reason that it is important to distinguish between the effects of marital status and transitions in marital status. If shifts in body weight associated with shifts in marital status are temporary, there may be little need for concern for policy relating marital status to health. If, however, marital status itself is more important than transitions in marital status, the policy implications are more likely to be of concern.

\section{Weaknesses in Prior Research}

The major weakness in prior literature is that the simultaneous effects of both marital status and transitions in marital status have not been considered. Research that has considered both marital status and transitions in marital status has modeled the effects of each separately (Averett et al., 2008; Umberson et al., 2009; Wilson, 2012). That is, separate models for marital status and transitions in marital status have been estimated, thus, allowing the effects of the two to be confounded. For example, Umberson et al. (2009) estimate one model in which the effects of being constantly never married, constantly divorced, and constantly widowed are compared with being constantly married. This model fails to consider the effects of marital transitions. In a second model, they estimate the effects of a transition from being unmarried to being married compared with respondents who remain continuously unmarried. And, in a third model, they compare the effects of a shift from being married to being widowed compared with being continuously married. Both the second and third models focus on transitions while ignoring marital statuses. Similar modeling choices are made by Averett et al. (2008) and Wilson (2012).

The research by Umberson et al. (2009) also represents confusion about the theoretical distinctiveness of the effects of marital status versus that of transitions in marital status. Take for example, the finding in their third model that becoming widowed between Time 2 and Time 3 affects both Time 3 body weight and Time 4 body weight. In their study, Time 2 corresponds to 1989, Time 3 to 1994, and Time 4 to 2001. Thus, their results imply that a transition to widowhood between 1989 and 1994 affects body weight for up to a minimum of 7 years. Such a long-term shift in body weight is not consistent with the transitory effects of the crisis model. Rather, it is more consistent with the more long-term effects expected for marital status (here, being widowed). 
But, the effects of marital status are not considered in this third model. It is possible, therefore, that the effect that they attribute to a transition in marital status is confounded with marital status itself. If the crisis model is to be supported, then there should be decay in the effect of a marital transition that should alter the baseline effect of the marital status that is the ending status of the transition. For example, the transition to widowhood should evidence itself in an effect that increases the baseline effect of being widowed itself but that decays over time. Such an effect can only be determined if the effects of both marital status and transitions in marital status are modeled simultaneously, and prior research has failed to accomplish this task.

\section{Data and Method}

\section{Data}

I use data taken from the 1979 NLSY. Survey respondents $(N=12,686)$ were aged 14 to 21 years in 1979 when they were initially interviewed. Follow-up surveys were administered annually through 1994, biennially thereafter. Information on height and weight were asked in numerous years of the survey. The dependent variable used in all analyses in this article is BMI, calculated as weight divided by the square of height in inches and then multiplied by 703 . I make use of information on weight collected in 1981, 1989, 1996, and 2004, or BMI assessed at approximately 8 -year intervals to minimize minor, random variations in BMI that might occur, measured at shorter intervals. ${ }^{1}$ Firebaugh and Beck (1994) note that when intervals are small and the number of resulting transitions is small, observed changes may be due to transient fluctuation and measurement error increases substantially. This is the case with marital transitions, which generally occur at a slow pace across time.

Information on height was taken from the 1985 survey, when the youngest respondents were aged 20 years. Furthermore, I focus on young men and women who were never married and aged between 16 and 19 years in 1979. In essence, I focus on how marital status and marital transitions influence BMI in a cohort of young, single respondents as they age from adolescence to middle age (age 39-42 years). For this cohort, I have full information on all transitions experienced by respondents; no respondents are observed after a marital status transition has occurred. The final sample size is 4,127 respondents of which 3,347 have full information on all variables used in this analysis.

Marital status is a time-varying (within-person) covariate that includes the following statuses measured at each of the four time points $(1981,1989,1996$, 2004); married (baseline or omitted category), single (never married), divorced, or cohabiting. There were an insufficient number of marriages terminated by 
death to include widowed as a category of marital status. Marital transitions is also a time-varying covariate that includes the following transitions: no transition (baseline or omitted category), transition out of marriage, transition to marriage, transition to cohabitation, transition out of cohabitation. Because all individuals are single in 1981, this variable refers to transitions that occurred in the intervals 1981-1989, 1989-1996, and 1996-2004. ${ }^{2}$ Time-varying control variables measured at each interval include log of family income (in constant 1984 dollars) and highest grade of education completed.

Between-person variation in body weight is modeled as a function of both gender and race (measured as White, Black, or Hispanic, with White constituting the baseline). A variety of research has indicated that both gender (Shafer, 2010; Umberson et al., 2009) and race (Kahn \& Williamson, 1991; Shafer, 2010; Umberson et al., 2009) are related to trajectories of body weight. Accordingly, estimates of the effects of marital status and transitions in marital status on body weight are net of race and gender effects on trajectories of body weight. I also test whether gender moderates the link between $\mathrm{BMI}$ and marital status and marital transitions.

\section{Statistical Model}

I examine trajectories of body weight using an LGCM. LGCMs are appropriate when the outcome variable being considered, here BMI, follows a trajectory of change across time that is not random. Conventional random-effects models (REMs) or fixed-effects models (FEMs) used to examine data with more than one observation per respondent generally ignore any patterned change in BMI over time, and most Americans exhibit a pattern of weight gain as they age (Ogden, Fryar, Carroll, \& Flegal, 2004). If there is an underlying trajectory of change in BMI then fit to the data should improve if it is modeled. One consequence is better estimates of the effects of the time-varying covariates once the effects of change have been taken into account. Traditionally LGCMs have been estimated as an extension of the REM framework by accounting for the systemic pattern of change in body weight over time. In such models, however, the terms for the latent trajectory are assumed to be independent of the time-varying covariates (e.g., Umberson et al., 2009). I extend the traditional LGCM by placing it within a fixed-effects framework that allows the terms for the latent trajectory of BMI to be correlated with the time-varying measures of marital status and changes in marital status. The use of fixed-effects estimators allows for some control for selectivity that is associated with unobserved fixed characteristics (e.g., unobserved fixed characteristics that are linked to marital status, transitions in marital status, and body weight). 
Consider the following simple REM:

$$
\mathrm{Y}_{i t}=\alpha_{t}+\boldsymbol{\beta}_{Y X} \mathbf{X}_{i t}+\beta_{Y \eta} \eta_{i}+\varepsilon_{i t}
$$

where $Y_{i t}$ is the value of BMI for the $i$ th case at time $t ; \alpha_{t}$ is an intercept term at time $t ; \mathbf{X}_{i t}$ is a vector of time-varying covariates for the $i$ th case at time $t$; $\boldsymbol{\beta}_{Y X}$ is a vector of coefficients indicating the effects of $\mathbf{X}_{i t}$ on $Y_{i t} ; \eta_{i}$ is a scalar indicating all the latent time-constant factors affecting $Y_{i t} ; \boldsymbol{\beta}_{Y \eta}$ is a vector of coefficients linking the latent factor, $\eta_{i}$, to $Y_{i t}$ (in most instances, these values are set equal to 1.0 , thus, ignoring any potential changes over time in the effect of the latent factor); and $\varepsilon_{i t}$ is a random disturbance for the $i$ th case at time $t$ with $E\left(\varepsilon_{i t}\right)=0$ and $\mathrm{E}\left(\varepsilon_{i t}^{2}\right)=\sigma_{\varepsilon t}^{2}{ }^{3}$ It is also assumed that $\varepsilon_{i t}$ is uncorrelated with $\mathbf{X}_{i t}$, and $\eta_{i}$, that $\operatorname{COV}\left(\varepsilon_{i t}, \varepsilon_{i t}\right)=0$ for $t \neq s$, and that $\eta_{i}$ is uncorrelated with $\mathbf{X}_{i t}$. This is the default REM estimated by most software products in which the effects of the time-varying variables are constrained to be constant across time, as are the variances of the error terms. This is also the REM that many, if not most, researchers using longitudinal data report. The key assumption that makes this an REM is that $\eta_{i}$ is uncorrelated with $\mathbf{X}_{i t}$. This assumption means that the standard errors of the coefficients are adjusted for clustering but are not adjusted for unmeasured covariates that may be correlated with both the dependent variable and the covariates.

A simple LGCM within the REM framework can be expressed as follows:

$$
Y_{i t}=\boldsymbol{\beta}_{Y X} \mathbf{X}_{i t}+\beta_{Y \eta 0} \eta_{0 i}+\beta_{Y \eta 1} \eta_{1 i}+\varepsilon_{i t}
$$

where $\eta_{0 i}$ is a latent factor indicating initial values of BMI with slopes, $\beta_{Y \eta 0}$, constrained to equal 1 and $\eta_{1 i}$ is a second latent factor with fixed slopes, $\beta_{Y \eta 1}$, indicating change in BMI over time. ${ }^{4}$ All other terms and assumptions are defined as in Equation (1) with the extension that the latent factor is now represented by (at least) two terms. Most researchers call $\eta_{0 i}$ the intercept (beginning or initial value of the outcome) and $\eta_{1 i}$ the slope of the trajectory in the model (change across time from the initial value of the outcome). In an LGCM, therefore, there are two latent components rather than one as is the case in a traditional REM. Similar to the case in an REM one latent factor (intercept) describes a stable component across time (slopes are constrained to unity). It is the second latent factor (slope) that allows variation from this stable component over time and can be thought of as representing the rate of change across time. As written in Equation (2), there is a single slope term that allows for a linear change in the outcome (here BMI) across time. As indicated later, additional slope terms can be added to the model to capture nonlinear (e.g., quadratic) change across time. 
The LGCM can be extended to a fixed-effects framework in a straightforward manner. As illustrated by Bollen and Brand (2010) and Teachman, Duncan, Yeung, \& Levy (2001) the key to extending an REM to an FEM is to relax the assumption that there is no correlation between $\mathbf{X}_{i t}$ and the latent terms, $\eta_{0 i}$ and $\eta_{1 i}$. It is straightforward to allow these terms to covary in the covariance structure approach used to estimate LGCMs. It is also possible to estimate hybrid models mixing fixed- and random-effects by allowing a subset of the variables in $\mathbf{X}_{i t}$ to be correlated with one or more of the latent terms. The ability to allow correlation between the time-varying covariates in a model and the latent terms $\left(\eta_{0 i}\right.$ and $\left.\eta_{1 i}\right)$ is an important elaboration of the standard LGCM. In nonexperimental research strong theoretical guidance would be necessary to fully justify an a priori assumption of no correlation between the latent terms and the measured time-varying covariates. If this assumption is violated, and a fixed-effects alternative is not estimated, then the resulting parameter estimates may be biased. The fixed-effects version of the LGCM provides an alternative to making this a priori assumption.

Recognizing that the LGCM comprises both within- and between-respondent variation, it is also possible to make both $\eta_{0 i}$ and $\eta_{1 i}$ be functions of time-constant covariates (i.e., the latent terms do not vary within respondents but can vary between respondents). For example,

$$
\begin{gathered}
\mathrm{Y}_{i t}=\beta_{Y X} \mathrm{X}_{i t}+\beta_{Y \eta 0} \eta_{0 i}+\beta_{Y \eta 1} \eta_{1 i}+\varepsilon_{i t} \\
\eta_{0 i}=\alpha_{00}+\gamma_{\eta 0 z} \mathbf{Z}_{i}+\delta_{0 i} \\
\eta_{1 i}=\alpha_{10}+\gamma_{\eta 1 z} \mathbf{Z}_{i}+\delta_{1 i}
\end{gathered}
$$

where $\alpha_{00}$ and $\alpha_{10}$ are constant terms, $\gamma_{\eta 0 z}$ and $\gamma_{\eta 1 z}$ are vectors of slopes, $\mathbf{Z}_{i}$ is a vector of time-constant variables affecting the latent intercept and slope, $\delta_{0 i}$ and $\delta_{1 i}$ are error terms, and all other terms are as defined earlier. In my analysis, $\mathbf{Z}_{i}$ is initially composed of indicators of gender and race. ${ }^{5}$ Thus, the estimated effects of the time-varying variables are net of any variations in latent growth trajectories associated with gender and race.

\section{Results}

\section{Descriptive Statistics}

Descriptive Statistics for the sample are shown in Table 1. These statistics are based on the 3,347 respondents in the data with complete information on all 
Table I. Descriptive Statistics for the Sample of Respondents in the 1979 National Longitudinal Study of Youth: $N=3,347$.

\begin{tabular}{lcccc}
\hline & \multicolumn{5}{c}{ Year } \\
\cline { 2 - 5 } Variable & 1981 & 1989 & 1996 & 2004 \\
\hline Body mass index & $22.08(3.17)$ & $24.96(4.40)$ & $26.93(5.12)$ & $28.29(5.51)$ \\
Marital status & .93 & .33 & .20 & .14 \\
$\quad$ Single & .01 & .13 & .19 & .23 \\
Divorced & .01 & .09 & .09 & .07 \\
$\quad$ Cohabiting & .05 & .45 & .52 & .56 \\
$\quad$ Married & - & .35 & .17 & .10 \\
Marital status transitions & - & .12 & .12 & .10 \\
$\quad$ Not married to married & - & .08 & .06 & .04 \\
Married to not married & - & .03 & .06 & .04 \\
$\quad$ Not cohabiting to cohabiting & - & $12.81(2.24)$ & $13.08(2.41)$ & $13.30(2.45)$ \\
$\quad$ Cohabiting to not cohabiting & - & $26,745(92,712)$ & $26,507(63,837)$ & $27,623(33,244)$ \\
Control variables & - & & &
\end{tabular}

Note. Standard errors are in parentheses.

variables. It can be seen that BMI increases steadily from 1981 (22.08) to 2004 (28.29). Most of the sample is single in 1981 (93\%) and by 2004 the majority of the sample is married (56\%). There is substantial transition between marital statuses, with fewer transitions occurring after 1996. The most common transition is from being not married to married, followed by being married to not married. Education and family income follow the expected pattern of change across time. Transitions are not shown for the period 1979 to 1981 because very few transitions in marital status occurred during the period. Furthermore, variation in highest grade completed is sufficiently low in 1981 that models including this variable had difficulty in converging. Consequently, the effects of the control variables are only modeled from 1989 onward.

\section{Results for Latent Growth Curve Models}

Model fit statistics for several LGCMs are shown in Table 2. All models were estimated using PROC CALIS in SAS. I initially estimated models using a full information maximum likelihood (FIML) option that provides estimates using all cases $(N=4,127)$, including those with missing values. Unfortunately, the FIML option became increasingly difficult to reach convergence as model complexity increased. Thus, estimates shown in Table 2 are based on the 3,347 respondents in the database with full information on all variables. ${ }^{6}$ 
Table 2. Model Fit Statistics for Various Latent Growth Curve Models: NLSY Data on BMI.

\begin{tabular}{lccc}
\hline Model & LR $\chi^{2} / d f$ & RMSEA & BIC \\
\hline Combined models & & & \\
A. LGC REM & $1009.99 / 8$ & .194 & 943.39 \\
B. Model A + quadratic & $12.49 / 4$ & .025 & -20.81 \\
C. Model C + marital status + transitions & $141.45 / 93$ & .013 & -633.80 \\
D. Model D + lagged transitions & $136.65 / 84$ & .014 & -562.68 \\
E. FEM LGC + quadratic + marital status + transitions & $238.15 / 59$ & .030 & -253.05 \\
F. Hybrid LGC + quadratic + marital status + transitions & $130.17 / 89$ & .012 & -610.78 \\
G. Model B + controls & $273.60 / 119$ & .020 & -717.11 \\
H. Model G + mediated effects of race/ethnicity and & $272.54 / 122$ & .019 & -743.15 \\
$\quad$ gender & & & \\
Models estimated by gender & $1237.97 / 187$ & .058 & -318.86 \\
I. Model H + no constraints by gender & $918.32 / 196$ & .047 & -713.44 \\
J. Model I + equality constraints for error terms and & & & \\
$\quad$ covariances & $876.20 / 201$ & .045 & -797.19 \\
K. Model J equality constraints for slopes of time- & & & \\
$\quad$ varying variables & & & \\
\hline
\end{tabular}

Note. NLSY = National Longitudinal Survey of Youth; FEM = fixed-effects model; REM = random-effects model; $\mathrm{BMI}$ = body mass index; LR, likelihood ration; RMSEA = root mean square error of approximation; $\mathrm{BIC}=$ Bayesian information criterion.

Model A fits a simple LGCM corresponding to Equation (2) with no covariates (e.g., a model within the REM framework). Three indicators of model fit are shown, including the likelihood ratio (LR) $\chi^{2}$, root mean square error of approximation (RMSEA), and Bayesian information criterion (BIC). Well-fitting models should have a value less than .1 for the RMSEA and negative values of BIC (Bollen \& Brand, 2010). If models are nested, then differences between the respective LR $\chi^{2}$ values can be used as an indicator of change in model fit. It is apparent that Model A does not fit the data well. Model B enhances Model A by adding a quadratic term to the model. ${ }^{7}$ The result is a substantial improvement in model fit. RMSEA drops considerably and the BIC value is now negative. Model B thus indicates that BMI changes across time but not in a linear fashion.

Model $\mathrm{C}$ adds the effects of marital status and transitions in marital status in the prior interval. Once again, model fit improves substantially. In particular, BIC now takes a much more negative value. In Model $\mathrm{C}$ the effects of transitions are restricted to those that occurred in the prior interval. Transitions that occurred earlier are assumed to have no effect on BMI. This assumption is consistent with the crisis model. Model D relaxes this assumption and allows transitions in prior intervals to affect BMI. The results from estimating this model indicate deterioration in model fit (RMSEA is larger and BIC is 
substantially less negative). This pattern indicates that lagged transitions do not play an important role in determining BMI, again consistent with the crisis model. That is, the effects of transitions in marital status are relatively short-lived (in this case, they do not expand beyond the 8-year gap between measures of BMI). Thus, there is support for the notion that both marital status and transitions in marital status have an effect on body weight.

The models estimated so far are all REMs. That is, they assume that the latent terms included in the model are all independent of the included timevarying covariates. As indicated earlier, this is a strong assumption. Model $\mathrm{E}$ tests this assumption by allowing nonzero covariances between each of the latent terms and each of the measures of marital status and transitions between marital statuses in Model C. The model fit statistics indicate that this model does not fit the data as well as the corresponding REM (Model C). In particular, the BIC value is substantially less significant. This is not an unexpected result, however, because the BIC statistic substantially penalizes less parsimonious models (Bollen \& Bland, 2010); and by estimating a substantial number of additional covariances, Model E (not shown) is much less parsimonious than Model C. It is possible, however, that only a subset of the potential covariances involved in the full FEM version of the LGCM are statistically different from zero and can be estimated without unduly affecting the BIC. An examination of the covariances estimated in Model E indicated that only those between the first latent term $\left(\eta_{0 i}\right)$ representing the baseline value of BMI and the time-varying variable measuring whether the respondent was single were consistently different from zero. Accordingly, I estimated Model F, which is a hybrid LGCM setting all covariances between the latent terms and time-varying covariates to equal zero except those involving $\eta_{0 i}$ and the indicators of being single. This model fits much better than the full FEM represented by Model E. When compared with the REM version in Model C, the hybrid model fits better according to the difference between the two LR $\chi^{2}$ values $(11.28,4$ $d f$ ) and the RMSEA (.013 vs. .012) ${ }^{8}$ However, the BIC value for Model $\mathrm{F}$ is not as negative as for Model C. Thus, there is some ambiguity as to which is the best fitting model. I elected to retain Model F because of the consistently nonzero estimates of the covariances between $\eta_{0 i}$ and the time-varying measure of being single.

The next model estimated, Model G, includes the effects of the two timevarying control variables, education and log of family income. According to the BIC statistic (but not the RMSEA), this model fits the data better than Model F. Model $\mathrm{H}$ allows the latent terms (the $\eta$ ) to be functions of race and gender. Again, according to BIC, this model fits the data better than Models $\mathrm{F}$ and $\mathrm{G}$. 
I assume that Model $\mathrm{H}$ is the best fitting model and provide parameter estimates for this model in Table 3. These estimates show which marital statuses and transitions in marital status have substantive impact on body weight. The estimates show that single and divorced respondents weigh about .53 to .56 lower on the BMI scale than comparable married respondents (a difference of about 3.2 pounds for someone 70 inches tall and about 150 pounds). Cohabiting respondents do not differ from married respondents with respect to BMI. Tellingly, in terms of transitions, only respondents who transition out of marriage weigh less, about .23 on the BMI scale (about 3.1 pounds for someone 70 inches tall and about 150 pounds). Transitions into marriage are not linked to body weight.

Although the only transition to affect BMI is a transition out of marriage, some caution should be taken in interpreting these results. First, because the period between measurements covers 8 years, the estimates involve a mixture of transitions that occur over a relatively wide range of time. That is, in any given interval there may be up to 8 years difference in the timing of a transition. Thus, the extent to which the effect of a transition is transitory, the estimated effects shown in Table 3 are likely to be conservative. On the other hand, as suggested earlier, truly short-term effects on body weight may be of little consequence theoretically or practically. Second, some transitions within the categories used may not be of equal importance. For example, a transition out of cohabitation to marriage may have an effect different from a transition out of cohabitation to living alone. Only larger sample sizes, those with more transitions, will allow researchers to sort these issues.

The average initial BMI in the data is 21.489 for White women. Blacks (0.384), Hispanics (0.350), and men (1.080) all weigh more when they are adolescents. The slope for White women is 2.204 and is higher for Blacks (0.876), Hispanics (0.635), and men (1.008). The quadratic term indicates a decrease in the rate of growth in BMI over time for all groups, with the decrease being more substantial for Blacks, Hispanics, and men. The latent terms for the trajectory of change in BMI over time thus indicate substantial increase in body weight over time but at a decreasing pace. For example, a single White woman 66 inches tall and weighing 130 pounds in 1981 would be expected to weigh 142 pounds in 1989, 153 pounds in 1996, and 161 pounds in 2004.

There remains significant residual error in BMI after accounting for marital status and the control variables. ${ }^{9}$ Similarly, there remains significant residual variation in the latent terms after accounting for the effects of race and gender. There is also a positive covariance between the latent intercept term and the latent slope, indicating that individuals who weigh more initially are also more likely to gain weight rapidly, although the covariance between the 
Table 3. Estimated Components of Various Latent Growth Curve Models: NLSY Data on BMI.

\begin{tabular}{|c|c|c|c|}
\hline \multirow[b]{2}{*}{ Model Components } & \multirow[b]{2}{*}{ Model $\mathrm{H}$} & \multicolumn{2}{|c|}{ Model K } \\
\hline & & Men & Women \\
\hline \multicolumn{4}{|l|}{ Manifest variable equations } \\
\hline Single & $-.557 * *$ & $-.722 * *$ & $-.722 * *$ \\
\hline Divorced & $-.525 * *$ & $-.519 * *$ & $-.519 * *$ \\
\hline Cohabiting & -.130 & -.066 & -.066 \\
\hline Transition out of marriage & $-.227^{*}$ & $-.287 * *$ & $-.287^{* *}$ \\
\hline Transition to marriage & .005 & -.098 & -.098 \\
\hline Transition to cohabitation & .231 & -.344 & -.344 \\
\hline Transition out of cohabitation & -.005 & .122 & .122 \\
\hline Highest grade completed & $.038^{*}$ & $.059 * *$ & $.059 * *$ \\
\hline Log of family income & .001 & $.038^{* *}$ & $.038^{* *}$ \\
\hline \multicolumn{4}{|l|}{ Latent variable equations } \\
\hline Intercept & $21.489 * *$ & $23.347^{* *}$ & $21.753 * *$ \\
\hline Black & $.384 * *$ & $-.454^{* *}$ & $1.216^{* *}$ \\
\hline Hispanic & $.350 * *$ & .257 & $.402 * *$ \\
\hline Male & $1.080 * *$ & - & - \\
\hline Slope & $2.204^{* *}$ & $\mathrm{I} .54 \mathrm{I} * *$ & -.049 \\
\hline Black & $.876 * *$ & $.738 * *$ & $2.364 * *$ \\
\hline Hispanic & $.635^{* *}$ & $.812^{* *}$ & $1.755^{* *}$ \\
\hline Male & $1.008 * *$ & - & - \\
\hline Quadratic slope & $-.177 * *$ & -.049 & $.381 * *$ \\
\hline Black & $-.08 I^{*}$ & -.089 & $-.445^{* *}$ \\
\hline Hispanic & $-.092 *$ & $-.145 * *$ & $-.393 * *$ \\
\hline Male & $-.181 * *$ & - & - \\
\hline Error BMII & $3.161 * *$ & $3.209 * *$ & $3.209 * *$ \\
\hline Error BMI2 & $3.161 * *$ & $3.209 * *$ & $3.209 * *$ \\
\hline Error BMI3 & $3.161 * *$ & $3.209 * *$ & $3.209 * *$ \\
\hline Error BMI4 & $3.161 * *$ & $3.209 * *$ & $3.209 * *$ \\
\hline Error intercept & $6.646 * *$ & $6.489 * *$ & $6.489 * *$ \\
\hline Error slope & $6.090 * *$ & $6.288 * *$ & $6.288 * *$ \\
\hline Error quadratic slope & $.398 * *$ & $.410 * *$ & $.410 * *$ \\
\hline \multicolumn{4}{|l|}{ Covariances } \\
\hline Intercept—slope & $3.162 * *$ & $3.108 * *$ & $3.108 * *$ \\
\hline Intercept-quadratic slope & $-.752 * *$ & $-.74 I * *$ & $-.74 I * *$ \\
\hline Slope-quadratic slope & $-1.358^{* *}$ & $-1.408 * *$ & $-1.408 * *$ \\
\hline Intercept-Time I single & .019 & .008 & .008 \\
\hline Intercept-Time 2 single & $.045 * *$ & $.029 * *$ & $.029 * *$ \\
\hline Intercept-Time 3 single & $.045 * *$ & $.027^{*}$ & $.027 *$ \\
\hline Intercept-Time 4 single & $.031 * *$ & .023 & .023 \\
\hline
\end{tabular}

Note. NLSY = National Longitudinal Survey of Youth; BMI = body mass index. 
intercept and the quadratic slope indicates that this more rapid pattern of growth slows over time. The negative covariance between the two slope terms suggests that higher slopes are more likely to have more negative quadratic terms. Finally, the covariances between the latent intercept term and being single are positive after 1981, indicating that single persons are more likely to weigh more than would be expected given the measured variables. Failure to take this term into account would yield an underestimate of the effect of being single on BMI and, thus, reduce the overall effects of all marital statuses.

Model $\mathrm{H}$ assumes that the effects of marital status and changes in marital status are equivalent for men and women. As indicated earlier, past research suggests that gender may moderate the impact of marital status on weight. To test this possibility, I first estimated a model with separate effects for men and women with no equality constraints. Conceptually, this model is equivalent to the separate models for men and women estimated in prior research. Model I in Table 2 indicates that this model does not fit the data well. In particular, the BIC value is substantially less negative than Model H. Clearly, a more parsimonious model is in order. Consequently, in Model J, I constrain error terms and covariances to be equal across gender but allow the effects of the latent terms and of the time-varying covariates (marital status and transitions in marital status) to vary across gender. This model fits the data better than Model I (BIC is -713.44) but still represents a poorer fit than Model H. The final model estimated, Model K, further constrains the effects of the timevarying covariates to be equal across gender, allowing only the effects of the latent terms to vary. This model fits the data well, and the BIC value (-797.19) indicates a better fit than Model $\mathrm{H}$. Thus, there appears to be no differences between men and women in the effects of marital status and transitions in marital status on body weight. This result is contrary to some of the prior research (Averett et al., 2008; Sobal et al., 2003; Wilson, 2012). Note, however, that the prior research has simply presented results for models estimated separately by gender without formally testing the statistical significance of any differences noted.

The parameter estimates for Model $\mathrm{K}$ are shown in Table 3. Because they are constrained, the effects of marital status and changes in marital status are identical for men and women, providing no support for the notion that gender moderates the effects of marital status on BMI. The coefficients shown for Model $\mathrm{K}$ indicate the same story for the effects of marital status and changes in marital status as indicated in Model $\mathrm{H}$ (with the exception that the effect of being single is now somewhat stronger).

Differences between men and women appear only in the latent terms and the effects of race on these latent terms. Men have higher initial levels of BMI 
and their weight generally increases in a linear fashion across time (although there is evidence for a quadratic pattern for Hispanic men). For women, change in body weight depends strongly on race. For White women, there is a small, positive quadratic change across time. For Blacks and Hispanics, there is a much stronger linear increase across time.

\section{Illustrating the Effects of Marital Status and Marital Transitions}

Using values shown for Model K, Figure 1 graphically represents change in BMI across time for continuously married respondents according to race/ ethnicity and gender. For White men and Hispanic men, body weight increases rapidly from 1981 to 1989 and then continues to increase at a slower pace through 2004. For Black men, weight is relatively stable from 1981 to 1989, increases very rapidly from 1989 to 1996, and then continues to increase at a slower pace through 2004. Black women experience the most rapid increase in body weight, as well as the greatest absolute increase in body weight. Hispanic women experience a rapid gain in weight between 1981 and 1989, and then less rapid increases thereafter. White women experience the slowest growth in body weight across time and gain the least in terms of absolute body weight. Figure 2 shows the same pattern of change across time for continuously single respondents. The curves are similar to those for continuously married individuals, but the starting and ending weights are smaller, representing the negative effect of being single on BMI.

Figure 3 shows the effect of divorce on body weight. The values shown are for White men and represent BMI values for a man who divorced in the 1981-1989 interval compared with a man who remained continuously married. The gap between the two groups is greatest in 1989 when the effect of making the transition out of marriage is added to the effect of being single. Over time, however, the gap between the two groups lessens (but does not converge) as the temporary effect of a divorce wanes. Similar curves for other groups defined by race and gender will follow somewhat different paths (as indicated by the paths shown in Figures 1 and 2) but the shift in weight associated with a divorce will show the same pattern of differences between married and divorced respondents demonstrated in Figure 3.

\section{Discussion}

The results presented in this article indicate that both marital status and transitions between marital statuses affect body weight. Married respondents are heavier than either never-married or divorced respondents. There is no difference in body weight between cohabitors and married respondents. Perhaps 


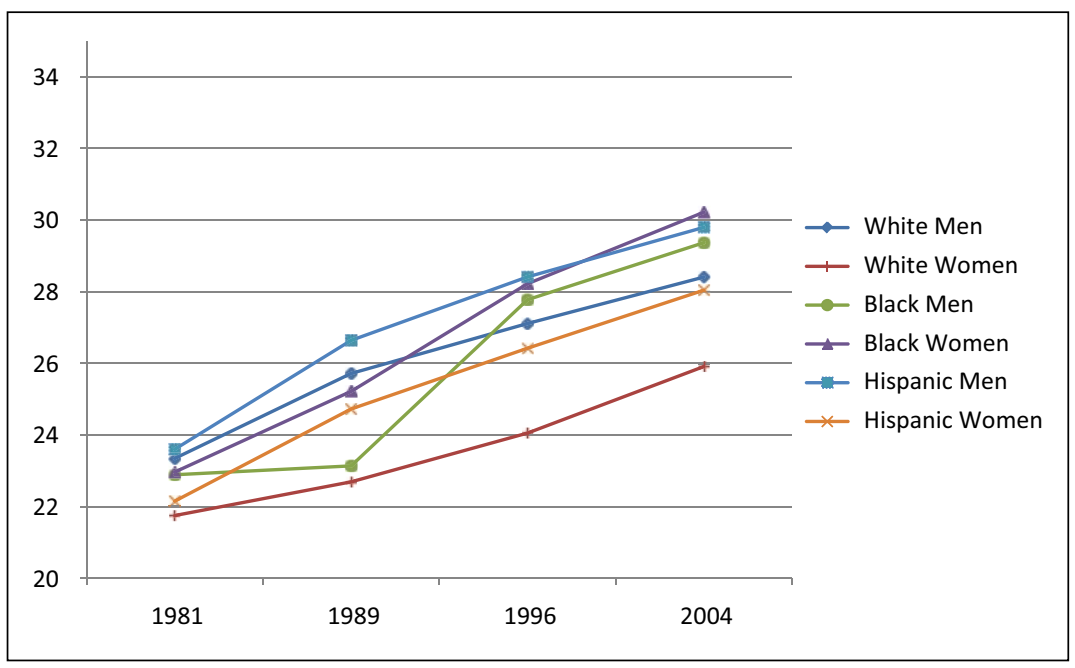

Figure I. Body mass index (BMI) of continuously married respondents: 198I-2004.

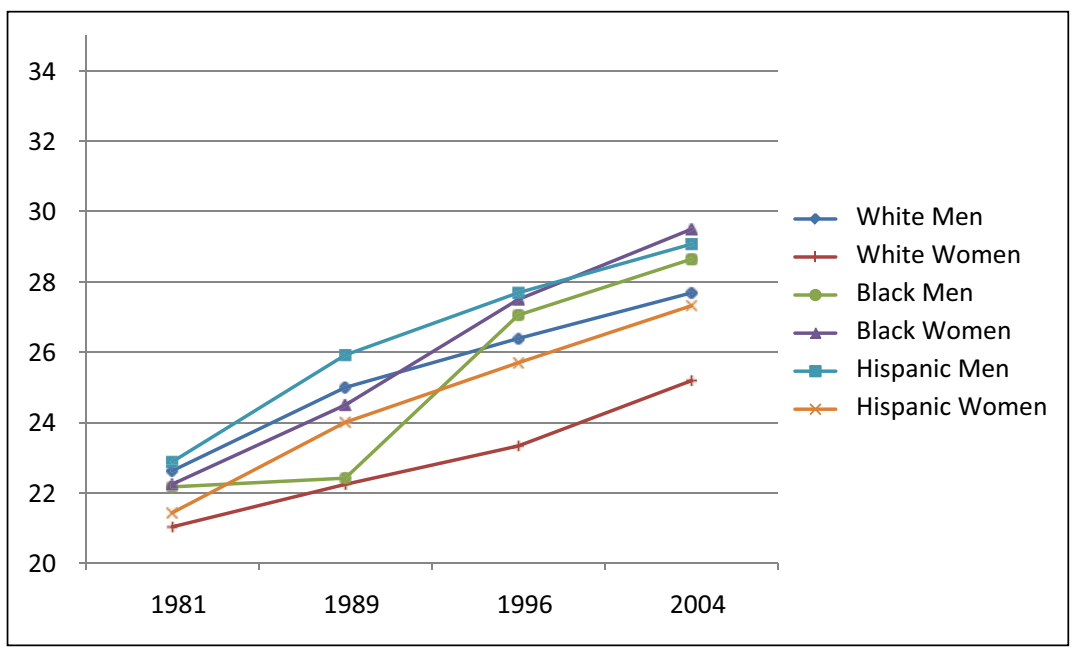

Figure 2. Body mass index (BMI) of continuously single respondents: 1981-2004.

most important, only one marital transition was found to affect body weight; respondents who divorced experienced a decline in body weight, and no other transition evidenced an effect on body weight. As expected, this effect 


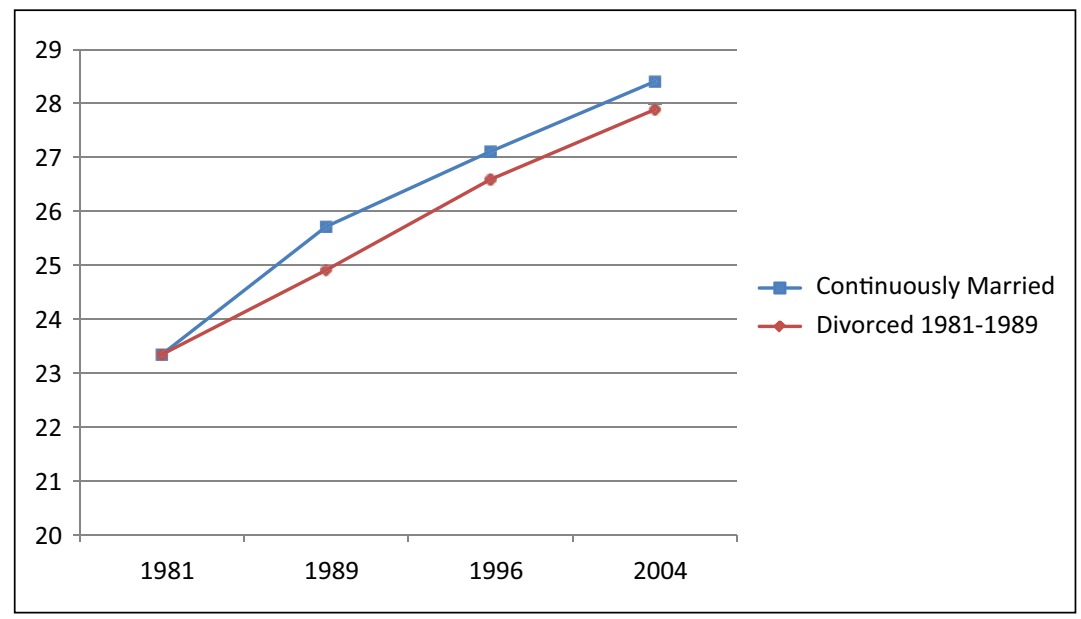

Figure 3. Body mass index (BMI) of continuously married White Men compared with White men who divorced 198I-1989.

is transitory, and fails to affect body weight in the long term. The results thus provide support for multiple sources of influence on body weight. The crisis perspective is supported by the temporary effect of divorce on BMI. The resource and attractiveness models are supported by the positive effect of marriage on BMI status. Unlike prior studies, these results are based on models that simultaneously control for the effects of marital status and transitions in marital status.

The effects of marital status and transitions in marital status are conditioned on a latent trajectory of weight gain across time. That is, the effects shown are all net of any underlying tendency for Americans, as represented in this sample, to gain weight as they age. The estimates are also free of any unmeasured latent factors that may be tied to both body weight and marital status and which helps reduce the likelihood that results may be due to selectivity. Indeed, the hybrid model suggests that being single is potentially linked (negatively) to the underlying trajectory of weight gain.

The results also indicate that the relationship between BMI and both marital status and transitions in marital status is not contingent on gender. Results suggest that the estimated effects of these variables are identical for men and women. This result differs from the findings of prior research. I use a global test for differences according to gender, whereas prior research has tended to use differences between estimates for men and women derived from separate models but not tested for statistical significance. The results presented in this 
article do not mean that there are no differences between men and women in BMI over time. Men tend to be heavier but gain weight less rapidly than women, although this is further conditioned by race. In particular White women, experience the least rapid gain in weight, whereas Black women experience the most rapid weight gain.

Before concluding, it is important to note a number of weaknesses in this analysis. First, even though both current marital status and marital transitions are considered, transitions could have occurred anywhere in an 8-year span. The extent to which the timing of transitions is important, especially if there is decay in the effects of a transition over time, will lead to bias in effects presented. Thus, some short-term results may be underrepresented, but, as mentioned earlier, such short-term changes in body weight are not likely to be of substantive interest in linking body weight to health consequences.

Second, the results shown are limited to a slice of historical time over the relatively early life course of adults. Results may differ in a different historical context. They may also differ for different parts of the life course. For example, for older respondents, widowhood may be a status or transition that is important to weight change. Third, transitions are not distinguished according to origin or destination status. Subsequent research with larger samples should strive to parse differences in the effects of transitions that are measured in finer detail.

Overall, the results indicate that marital status is more important for determining differences in body weight than transitions in marital status. This finding makes sense theoretically, in that the crisis model that supports the effect of transitions in marital status on body weight supposes that relationship will be transitory. Indeed, the emphasis on marital transition in prior literature may be misplaced. As shown earlier, not only are the effects of transitions limited to a small subset of all transitions (the transition to divorce), but the effects of transitions are short-lived. This is not to say that the effects of transitions should not be modeled, if only to remove their transitory effects from more stable effects of marital status.

In terms of policy, one can question whether the differences in body weight identified in this article are substantial enough to warrant public concern. Marital status differences in body weight are on the order of about 3 pounds at any point in time, a difference that is relatively small when compared with the latent growth in body weight as individuals age. Note, however, that the respondents in the NLSY79 are still young. At the end of this study, respondents were only aged 39 to 42 years. Differences in body weight according to marital status may be more substantial as individuals continue to age. Moreover, the effect of a 3-pound difference in body weight may depend on where in the continuum of BMI the difference occurs. At more healthy 
levels of body weight, say below 30 on the BMI scale, a difference of 3 pounds may not be consequential. At higher body weights, each additional pound may be more detrimental, and by 2004 in the NLSY79, a gain of 3 pounds would push the average respondent into the category of being obese.

The finding that men and women do not respond differently to marital status and changes in marital status suggests that prior research has likely overstated gender differences in the effects of marital status. Certainly, men and women have different latent trajectories in body weight, but these different trajectories are not the consequences of any moderating effects of gender. Rather, men and women appear to react to marital status and changes in marital status in similar fashion. Thus, assumptions such as women being more sensitive to changes in marital status due to gendered differences in the value of physical attractiveness are not supported. The results reported suggest that other factors must account for the observed gender differences. One such factor identified in this article is race, with Black women gaining weight much more rapidly than White women.

\section{Conclusion}

Using data taken from the 1979 NLSY, I have demonstrated that marital status affects body weight. In particular, living without a partner, either being divorced or never married, is associated with lower body weight. Cohabitors and married respondents tend to weigh more. Marital transitions also matter but only for divorce. Respondents who divorce experience a short-term decline in BMI. The results indicate that gender does not moderate the impact of marital status and transitions in marital status on body weight.

Subsequent research should continue to seek explain the effect of marital status and transitions on body weight. The NLSY data provide little in the way of explanatory variables. Thus, it is not possible to adjudicate between the resource and attractiveness models of weight change with any degree of specificity (although the results shown are net of the effects of two important resource variables, education and income).

\section{Declaration of Conflicting Interests}

The author(s) declared no potential conflicts of interest with respect to the research, authorship, and/or publication of this article.

\section{Funding}

The author(s) received no financial support for the research, authorship, and/or publication of this article. 


\section{Notes}

1. It is possible that self-reported weight is subject to bias. However, some evidence suggests high reliability in self-reported height and weight measures (Brener, McManus, Galuska, Lowry, \& Weschler, 2003). Moreover, because I focus on longitudinal measures of BMI, consistent misreporting of weight across time should not affect estimates.

2. For a small number of cases, more than one transition occurred in an interval. In those cases, I measured a transition as the difference between marital status at time $t$ and marital status at time $t-1$. Sample size was insufficient to separate transitions according to specific origins or destinations (e.g., a transition to marriage from single vs. a transition to marriage from cohabitation). Despite this limitation, all transitions out of marriage involve a separation or divorce and all transitions into a marriage involve a marriage; and these encompass the vast majority of all transitions.

3. Although it is not shown, the REM can also include time-constant covariates by assuming that they are uncorrelated with the latent term $\eta_{i}$.

4. In this current analysis, the fixed slopes are set to equal to $0,1,2$, and 3 , respectively. Thus, the slopes indicate change in BMI per 8-year period.

5. Umberson et al. (2009) use an LGCM similar to the one used in my analysis. There are three major differences, however. First, I use a fixed-effects version of the LGCM whereas they used the traditional random-effects version. Second, I consider the role of cohabitation in determining body weight. Third, I treat marital status as a wholly time-varying covariate. Umberson et al. treated marital status as both a time-constant and a time-varying variable. Respondents who did not experience a marital transition over the four waves of data in their data set were used to constitute time-constant variables affecting the latent intercept and latent slope. Respondents who experienced marital transitions were used to construct time-varying variables allowed to directly affect body weight.

6. FIML estimates of parameters were available for Models A through F in Table 2. I compared the FIML estimates of parameters with those obtained using the sample without missing values. In no case was there a difference in either statistical significance or a substantive difference in estimated parameters. These comparisons lead me to believe that the use of the sample without missing values does not substantially bias estimated parameter estimates and their standard errors in the models shown in Tables 2 and 3.

7. This third latent term has fixed slopes equal to $0,1,4$, and 9 , or the square of the slopes for the linear term. The sign of this third latent term determines whether the rate of change in BMI is increasing or decreasing.

8. Model fit in this instance can be assessed using the difference between the two LR $\chi^{2}$ values because Model C is nested within Model F.

9. These values make more sense when compared with the residual variance when only the latent growth terms are considered (e.g., prior to including marital status and the control variables). Doing so indicates that marital status and the control variable reduce the residual variance by about $2 \%$ (results not shown). 


\section{References}

Averett, S., Argys, L., \& Sorkin, J. (2013). In sickness and in health: An examination of relationship status and health using data from the Canadian national public health survey. Review of Economics of the Household, 11, 599-633.

Averett, S., Sikora, A., \& Argys, L. (2008). For better for worse: Relationship status and body mass index. Economics \& Human Biology, 6, 330-349.

Averett, S., \& Korenman, S. (1996). The economic reality of the beauty myth. Journal of Human Resources, 31, 304-330.

Averett, S., \& Korenman, S. (1999). Black-white differences in social economic consequences of obesity. International Journal of Obesity, 23, 166-173.

Bollen, K., \& Brand, J. (2010). A general panel model with random and fixed effects: A structural equations approach. Social Forces, 89, 1-34.

Bray, G. (2004). Medical consequences of obesity. Journal of Clinical Endocrinology \& Metabolism, 89, 2583-2589.

Breeze, E., Clarke, R., Shipley, M., Marmot, M., \& Fletcher, A. (2006). Causespecific mortality in old age in relation to body mass index in middle age and in old age: Follow-up of the Whitehall cohort of male civil servants. International Journal of Epidemiology, 35, 169-175.

Brener, N., McManus, T., Galuska, D., Lowry, R., \& Weschler, H. (2003). Reliability and validity of self-reported height and weight among high school students. Journal of Adolescent Health, 32, 281-287.

Carmalt, J., Cawley, J., Joyner, K., \& Sobal, J. (2008). Body weight and matching with a physically attractive romantic partner. Journal of Marriage and Family, 70, 1287-1296.

Conley, D., \& Glauber, R. (2005). Gender, body mass and economic status (NBER Working Paper No. 11343). Retrieved from http://www.nber.org/papers/w11343. pdf

Ferraro, K., \& Kelley-Moore, J. (2003). Cumulative disadvantage and health: Longterm consequences of obesity? American Sociological Review, 68, 707-729.

Firebaugh, G., \& Beck, F. (1994). Does economic growth benefit the masses? Growth, dependence, and welfare in the Third World. American Sociological Review, 49, 631-653.

Flegal, K., Troianao, R., Pamuk, E., Kuczmarski, R., \& Campbell, S. (1995). The influence of smoking on the prevalence of overweight in the United States. New England Journal of Medicine, 333, 1165-1170.

French, S., Jeffrey, R., Forster, J., McGovern, P., Kelder, S., \& Baxter, J. (1994). Predictors of weight change over two years among a population of working adults: The Healthy Worker Project. International Journal of Obesity and Related Metabolic Disorders, 18, 145-154.

Greeno, C., \& Weng, R. (1994). Stress-induced eating. Psychological Bulletin, 115, 444-464.

Hahn, B. (1993). Marital status and women's health: The effect of economic marital acquisitions. Journal of Marriage and the Family, 55, 495-504. 
Harris, K. M., Less, H., \& DeLeone, F. (2010). Marriage and health in the transition to adulthood: Evidence for African Americans in the Add Health Study. Journal of Family Issues, 31, 1106-1143.

Hill, J. O., Wyatt, H. R., Reed, G. W., \& Peters, J. C. (2003). Obesity and the environment: Where do we go from here? Science, 299, 853-855.

Jeffrey, R., \& Rick, A. (2002). Cross-sectional and longitudinal associations between body mass index and marriage-related factors. Obesity Research, 10, 809-815.

Kahn, H., \& Williamson, D. (1990). The contributions of income, education, and changing marital status to weight change among U.S. men. International Journal of Obesity, 14, 1057-1068.

Kahn, H., \& Williamson, D. (1991). Is race associated with weight change in US adults after adjustment for income, education, and marital factors. American Journal of Clinical Nutrition, 53, 1566S-1570S.

Meltzer, A., \& Everhart, J. (1995). Self-reported substantial 1-year weight change among men and women in the United States. Obesity Research, 3(Suppl. 2), 123S-134S.

Must, A., Spadano, J., Coakley, E. H., Filed, A. E., Colditz, G., \& Dietz, W. H. (1999). The disease burden associated with overweight and obesity. Journal of the American Medical Association, 282, 1523-1529.

Ogden, C., Fryar, C., Carroll, M., \& Flegal, K. (2004, October 27). Mean body weight, height, and body mass index, United States 1960-2002. Advance Data From Vital and Health Statistics, No. 347. Hyatsville, MD: Centers for Disease Control, National Center for Health Statistics.

Rauschenbach, B., Sobal, J., \& Frangillo, E. (1995). The influence of change in marital status on weight change over one year. Obesity Research, 3, 319-327.

Shafer, E., (2010). The effect of marriage on weight gain and propensity to become obese in the African American community. Journal of Family Issues, 31, 1166-1182.

Sobal, J. (1991). Obesity and socioeconomic status: A framework for examining relationships between physical and social attributes. Medical Anthropology, 13, 231-247.

Sobal, J., Rauschenbach, B., \& Frongillo, E. (1992). Marital status, fatness, and obesity. Social Science \& Medicine, 35, 915-923.

Sobal, J., Rauschenbach, B., \& Frongillo, E. (2003). Marital status changes and body weight changes: A U.S. longitudinal analysis. Social Science \& Medicine, 56, 1543-1555.

Teachman, J., Duncan, D., Yeung, W. J., \& Levy, D. (2001). Covariance structure models for fixed and random effects. Sociological Methods \& Research, 30, 271-288.

The, N., \& Gordon-Larsen, P. (2009). Entry into romantic partnership is associated with obesity. Obesity, 17, 1441-1447.

Thompson, D., Edelsberg, J., Colditz, G., Bird, A., \& Oster, G. (1999). Lifetime health and economic consequences of obesity. Annals of Internal Medicine, 159, 2177-2183. 
Umberson, D. (1992). Gender, marital status, and the social control of health behavior. Social Science \& Medicine, 34, 907-917.

Umberson, D., Liu, H., \& Powers, D. (2009). Marital status, marital transitions, and body weight. Journal of Health and Social Behavior, 50, 327-343.

Waite, L. (1995). Does marriage matter? Demography, 32, 483-507.

Williams, K. (2003). Has the future of marriage arrived? A contemporary examination of gender, marriage, and psychological well-being. Journal of Health and Social Behavior, 44, 470-487.

Williams, K., \& Umberson, D. (2004). Marital status, marital transitions, and health: A gendered life course perspective. Journal of Health and Social Behavior, 45, 81-98.

Wilson, S. (2012). Marriage, gender, and obesity in later life. Economics \& Human Biology, 10, 431-453. 